\title{
EVOLUTION OF THE COLUMN FOULING PRODUCTION IN WELLS PRODUCING OIL END GAS: CASE STUDY
}

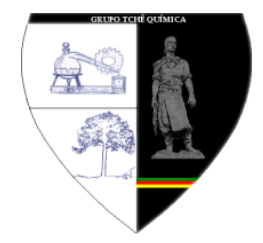

\author{
DA SILVA, Fernando Nunes 1; DA CUNHA, Jardel Dantas 2; BARBOSA, Andréa Francisca \\ Fernandes3 $\left(^{*}\right)$ e DA SILVA , Djalma Ribeiro4
}

\begin{abstract}
${ }^{1}$ Universidade Federal do Rio Grande do Norte, Programa de Pós Graduação em Engenharia Mecânica , Campus Universitário, Lagoa Nova, CEP 59078- 970, Natal-RN, Brasil

${ }^{2}$ Universidade Federal do Semi-Árido, Departamento de Ciência e Tecnologia Ambiental, Av. Francisco Mota, 572, bairro Costa e Silva, CEP 59625-900, Mossoró-RN, Brasil

${ }^{3}$ Universidade Federal do Maranhão, Bacharelado em Ciências e Tecnologia, Campus Balsas, José Leão, s/n CEP 65800000, Balsas-MA, Brasil

${ }^{4}$ Instituto de Química, Universidade Federal do Rio Grande do Norte, Av. Sem. Salgado Filho, s/n, Natal-RN, Brasil
\end{abstract}

*Autor Correspondente

*e-mail: andrea.barbosa@ufma.br

Received 14 April 2014; received in revised form 20 August 2014; accepted 21 August 2014

\section{RESUMO}

$\mathrm{Na}$ indústria petrolífera a problemática da formação de incrustação provoca uma série de danos, entre os quais podem ser citados a redução na produção de líquidos e consequentemente de petróleo, havendo também a elevação dos custos para a produção deste. O estudo das incrustações é então de suma importância para o conhecimento do mecanismo de sua formação e para a escolha do método de prevenção e/ou remoção. Técnicas de extração Soxhlet, EFRX, DRX e MEV, foram utilizadas para caracterização de uma amostra coletada na coluna de produção de um poço injetor de água. Através da análise dos resultados foi possível identificar: incrustação tipo ferruginosa, cuja sua formação está associada à presença de agentes corrosivos; e tipo carbonática, sendo sua formação influenciada pela concentração de íons de cálcio e bicarbonato, dissolvidos na água, uma vez que tal formação é comum em poços que apresentam teores elevados desses íns, que também é favorecida pela diminuição da pressão ou aumento da temperatura no reservatório.

Palavras-chave: Incrustação, corrosão, equipamentos, caracterização, coleta

\section{ABSTRACT}

In the oil industry the problem of scale formation causes a lot of damage, including the reduction in the production of liquid and thus can be cited oil, also with the increased costs for the production there of. The study of scale is then of paramount importance for the understanding of their formation mechanism and the choice of method for prevention and / or removal. Soxhlet extraction techniques EFRX, XRD and SEM were used to characterize a sample collected in the column of producing a water injection well. Through the analysis of the results was identified scale ferruginous type which its formation is associated with the presence of corrosive agents; and carbonate type, and its formation influenced by the concentration of calcium and bicarbonate ions dissolved in the water, since such formation is common in wells which have a high content of these ions, which is also favored by decreasing the pressure and temperature increase reservoir .

Keywords: Fouling, corrosion, equipment, characterization, collection 


\section{INTRODUÇÃO}

A medida que os campos produtores são exauridos, técnicas de estimulação como a injeção de água são implantadas com o objetivo de manter a pressão no interior do reservatório assim como também deslocar o óleo na direção dos poços produtores. Em virtude da incompatibilidade química entre a água de injeção rica em íons sulfatos $\left(\mathrm{SO}_{4}^{-2}\right)$, cloretos $\left(\mathrm{Cl}^{-}\right)$, bicarbonato $\left(\mathrm{HCO}_{3}^{-}\right)$e a água de formação rica em cátions como magnésio $\left(\mathrm{Mg}^{+2}\right)$, cálcio $\left(\mathrm{Ca}^{+2}\right)$, sódio $\left(\mathrm{Na}^{+2}\right)$, bário $\left(\mathrm{Ba}^{+2}\right)$ e estrôncio $\left(\mathrm{Sr}^{+2}\right)$ ocorre a precipitação de compostos inorgânicos denominados incrustações (DAHER, 2003).

Segundo MARQUES et al. (2001) as incrustações (ou scale) podem ser definidas como compostos químicos de natureza inorgânica que se encontram inicialmente solúveis em soluções salinas, e que precipitam podendo se acumular na formação, canhoneados, telas de gravel packing ${ }^{1}$, colunas de produção e equipamentos de superfície (WOOD, 2006). ATKINSON et al. (1997) diz que a formação de scale (incrustação) ocorre com a precipitação de minerais sólidos a partir de uma salmoura (água saturada).

Estas águas quando submetidas a condições termodinâmicas adequadas, faz com que sejam depositados compostos insolúveis, tais como $\mathrm{BaSO}_{4}$ (sulfato de bário ou Barita) (Equação 3.1), $\mathrm{SrSO}_{4}$ (sulfato de estrôncio ou Celestina) (Equação 3.2), $\mathrm{CaSO}_{4}$ (sulfato de cálcio ou Anidrita) (Equação 3.3) e $\mathrm{CaCO}_{3}$ (Carbonato de cálcio ou Calcita) (Equação 3.4) MARQUES et al. (2001). No caso da formação da calcita a mesma apresenta um mecanismo diferente que envolve um desequilíbrio termodinâmico entre o dióxido de carbono $\left(\mathrm{CO}_{2}\right)$ e o bicarbonato $\left(\mathrm{HCO}_{3}^{-}\right)$. Dentre estes compostos, o sulfato de bário é o mais problemático por ser o de menor solubilidade e o de mais difícil remoção (BOIM, 2003).

1Técnica para controle da produção de areia de formações com problemas de consolidação.

$$
\begin{aligned}
& \mathrm{Ba}^{+2}+\mathrm{SO}_{4}{ }^{2} \rightarrow \mathrm{BaSO}_{4} \downarrow \\
& \mathrm{Sr}^{+2}+\mathrm{SO}_{4}{ }^{2} \rightarrow \mathrm{SrSO}_{4} \downarrow \\
& \mathrm{Ca}^{+2}+\mathrm{SO}_{4}{ }^{2} \rightarrow \mathrm{CaSO}_{4} \downarrow \\
& \mathrm{Ca}^{+2}+2 \mathrm{HCO}_{3} \rightarrow \mathrm{CaCO}_{3} \downarrow+\mathrm{CO}_{2} \uparrow+\mathrm{H}_{2} \mathrm{O}
\end{aligned}
$$

Assim, no poço produtor, a pressão e temperatura diminuem ao longo da coluna até a superfície, mudando as condições termodinâmicas de precipitação nos componentes da coluna (RAMTAD, 2005).

Algumas incrustações de origem férrica em alguns casos, como nos que envolvem produtos de corrosão, como o óxido férrico $\left(\mathrm{Fe}_{2} \mathrm{O}_{3}\right)$, originando depósitos tipo incrustação (scalelike deposits) de natureza mista (lama argilosa com produtos de corrosão, por exemplo) (DEARBORN, 1991).

Ainda sobre o enxofre pode-se inferir que, quando o meio possui enxofre ou gases contendo esse elemento, como o gás sulfídrico $\left(\mathrm{H}_{2} \mathrm{~S}\right)$ e o dióxido de enxofre $\left(\mathrm{SO}_{2}\right)$, podem-se ter em temperaturas elevadas as possíveis reações, para o ferro (Equação 3.5, 3.6, 3.7 e 3.8) (GENTIL, 2007):

$$
\begin{gathered}
\mathrm{Fe}+\mathrm{S} \rightarrow \mathrm{FeS} \\
\mathrm{Fe}+\mathrm{H}_{2} \mathrm{~S} \rightarrow \mathrm{FeS}+\mathrm{H}_{2} \\
3 \mathrm{Fe}+\mathrm{SO}_{2} \rightarrow \mathrm{FeS}+2 \mathrm{FeO} \\
\mathrm{FeS}+2 \mathrm{SO}_{2} \rightarrow \mathrm{FeSO}_{4}+2 \mathrm{~S}
\end{gathered}
$$

Assim, no poço produtor, a pressão e temperatura diminuem ao longo da coluna até a superfície, mudando as condições termodinâmicas de precipitação nos componentes da coluna (RAMTAD, 2005). Desta forma as incrustações (ou scale) podem ser definidas como compostos químicos de natureza inorgânica que se encontram inicialmente solúveis em soluções salinas, as quais, quando submetidas a mudanças de pressão, temperatura e concentração mudam suas condições de solubilidade (DEMADIS, 2007). 
Com o objetivo de avaliar os produtos de incrustações formados e mostrar a importância das técnicas de caracterização o presente trabalho tem como objetivo analisar a composição das incrustações coletadas e sua morfologia.

\section{DESENVOLVIMENTO}

\section{Coleta e caracterização das amostras}

A amostra foi coletada durante a operação de intervenção na coluna de produção em um poço injetor de água sendo esta amostra denominada AM1.

A caracterização adequada dos resíduos de incrustação e/ou corrosão é uma informação bastante importante dado às formas de controle que podem ser implantadas tomando como base as informações obtidas de composição, morfologia e estrutura cristalina. Dentre as principais técnicas de caracterização usadas neste trabalho foram adotadas: a) Difração de raios- $X$, utilizada na caracterização das fases presentes, em um equipamento da marca Shimadzu modelo LabX XRD - 6000, com radiação CuKa $(\lambda=1,5418 \AA \hat{)})$. O difratograma obtido foi analisado pelos programas Search Match v.3.01 e Basic Process da Shimadzu com banco de dados do JCPDS (Joint Committee on Powder Diffraction Standards); b) Fluorescência de raios- $X$, aplicada na quantificação dos compostos presentes, em equipamento da marca Shimadzu modelo XRF-1800 no modo semiquantitativo no qual é empregada a metodologia de dispersão por comprimento de onda (WD$X R F)$. O equipamento possui limite de detecção do sódio ( $\mathrm{Na} \rightarrow Z=11)$ ao urânio $(U \rightarrow Z=92)$ utilizando radiação $\operatorname{RhKa}(\lambda=0,615 \AA \hat{A})$. Na preparação da amostra foi utilizada a prensagem da amostra com ácido bórico, utilizando uma proporção de 6:1 (ácido bórico: amostra) com dois ciclos de prensagem de 60 segundo com uma carga de $30 \mathrm{kPa}$; c) Microscopia eletrônica de varredura, aplicada no estudo da morfologia dos resíduos em um microscópio Philips modelo XL-30 ESEM em modo baixo vácuo (modo ambiental) e e) Extração Soxhlet em um equipamento Avanti modelo 2055-Soxtec, com copos e sistemas limpos e secos. Foi extraída $5 \mathrm{~g}$ de amostra, sendo representativa quando comparado a amostra total. O solvente utilizado no procedimento de extração foi clorofórmio, onde o mesmo é mais adequado por apresentar menor custo e baixa toxicidade. Foi utilizado 60 $\mathrm{ml}$ de clorofórmio, em que o ciclo de extração foi realizado a temperatura de $140{ }^{\circ} \mathrm{C}$ durante 120 minutos. Após extração, a amostra foi pulverizada em moinho planetário RETSCH PM $200 \mathrm{com} 500 \mathrm{rpm}$, durante 10 minutos, até amostra obter granulometria próxima de 100 \# (mesh) (MOURA, 2006).

\section{RESULTADOS E DISCUSSÃO:}

\section{Extração Soxhlet}

$\mathrm{Na}$ Tabela 01 são apresentados os resultados obtidos na extração Soxhlet para as amostras AM1. A fração orgânica foi 2,67\%, o que indica pouca ou nenhuma presença de hidrocarbonetos nas amostras em estudo.

Observa-se, na Figura 1a, que o resíduo coletado apresenta cor aparente marrom, indicando que há possibilidade da presença de ferro e que sua formação tenha se dada a partir de produtos corrosivos. A amostra já extraída e pulverizada (Figura1b) apresentou cor aparente semelhante à observada antes do processo de extração.

\section{Espectroscopia de Fluorescência de Raios-X (EFRX)}

O resultado de fluorescência (Tabela 02) apresentou como elementos majoritários: ferro (Fe-78,50\%), enxofre (S-18,36\%) e silício (Si$1,86 \%$ ) para a amostra AM1. A cor aparente marrom da amostra AM1 é devida à alta concentração de ferro na amostra. Através deste resultado também é possível inferir que a amostra AM1 se trata de um produto de corrosão formado na coluna de produção.

\section{Difratometria de Raios (DRX)}

Através da análise do difratograma (Figura 02) foram detectadas quatro fases cristalinas, as quais apresentaram segundo banco de dados JCPDS (Tabela 03) a seguinte classificação: greigita $\left(\mathrm{Fe}_{3} \mathrm{~S}_{4}\right)$, Goetita $\left(\mathrm{Fe}^{+3} \mathrm{O}(\mathrm{OH})\right)$, Mackinawita (FeS) e Smythita 
$\left(\mathrm{Fe}_{9} \mathrm{~S}_{11}\right)$. As fases presentes confirmam o alto teor de ferro e enxofre detectados pela técnica de EFRX (Tabela 3) e a sua formação esta associada à ação do $\mathrm{H}_{2} \mathrm{~S}$ com provável contaminação pelo oxigênio após a amostra ser retirada da coluna de produção.

A Goetita é resultado da oxidação superficial da amostra quando esta entra em contato com a atmosfera fora do poço. Devido a sua estabilidade, a Goetita é a fase predominante mais popularmente chamada "ferrugem".

Os produtos de corrosão originários da reação do $\mathrm{H}_{2} \mathrm{~S}$ na superfície do aço, podem se apresentar sob as mais diversas formas. O tipo de sulfeto de ferro depende de inúmeras variáveis como temperatura, salinidade, concentração de sulfeto presente, pressão parcial de $\mathrm{H}_{2} \mathrm{~S}, \mathrm{pH}$, presença de bactérias redutoras de sulfato e de outros gases como $\mathrm{CO}_{2}$ e $\mathrm{O}_{2}$. Produtos de corrosão de estruturas cúbicas como a greigita $\left(\mathrm{Fe}_{3} \mathrm{~S}_{4}\right)$ e as estruturas tipo ortorrômbicas são formadas pelo aumento na concentração dos íons enxofre (HEMMINGSEN, 1998).

Em sistemas dominados pelo $\mathrm{CO}_{2}$, a presença de pequenas quantidades de $\mathrm{H}_{2} \mathrm{~S}$ pode levar à formação de um depósito de sulfeto de ferro chamado Mackinawita, em temperaturas abaixo de $120^{\circ} \mathrm{C}$. Isto ocorrerá quando a razão entre a pressão parcial do $\mathrm{CO}_{2}$ e a pressão parcial do $\mathrm{H}_{2} \mathrm{~S}\left(\rho \mathrm{CO}_{2} / \rho \mathrm{H}_{2} \mathrm{~S}\right)$ for maior que 200. A mackinawita é um depósito fino, que reduz a taxa de corrosão e sua estabilidade é ainda desconhecida onde esta sofre transformações na presença de oxigênio gerando a greigita (HEMMINGSEN, 1998).

\section{Microscopia Eletrônica de Varredura (MEV)}

$\mathrm{Na}$ amostra AM1 (Figura 5-I) através da análise de MEV, utilizando-se os elétrons retroespalhados (BSE), os quais permitem visualizar que áreas apresentam elementos com maiores e menores pesos atômicos, foram analisados alguns pontos (A, B, C, D e E) por EDS. Tais pontos apresentaram composição química quase uniforme (devido a análise ser local) em todas as análises, com o ferro e o enxofre aparecendo em altas concentrações, estando, dessa forma, em conformidade com os resultados de EFRX e DRX apresentados anteriormente. Já na Figura 5-II, tem-se a imagem dos elétrons secundários (SE), os quais permitem visualizar a morfologia da amostra, que apresentou características superficiais semelhantes por toda a amostra, ou seja, sem variações na morfologia.

$\mathrm{Na}$ Figura 5 é mostrada a região que se encontrava em contato com o tubo metálico, onde é observada uma área de interface em "B". Conforme a Tabela 6, "A" apresentou um teor de enxofre $4,64 \%$, bem abaixo quando comparado com as demais regiões, indicando que essa região possivelmente é formada por óxidos e hidróxidos de ferro, como a Goetita encontrada através das análises de DRX. Essa corrosão possivelmente foi promovida pela presença do oxigênio. Com o possível aumento na concentração de $\mathrm{H}_{2} \mathrm{~S}$ na produção, acarretou a formação de outras camadas com teores de enxofre (B, C, D e E) mais elevados e, na amostra, presença das fases greigita, Mackinawita e Smythita em maior concentração.

O material incrustado na parede interna da coluna de produção (Amostra AM1) é uma incrustação do tipo ferruginosa, sendo esta resultante da ação corrosiva do oxigênio e do $\mathrm{H}_{2} \mathrm{~S}$, comumente encontrado em poços de produção, onde a sua origem está associada tanto à ação biogênica como à abiogênica (NEAL, 2001). A formação da calcita presente na amostra AM1, pode ter ocorrida devido à presença de íons de $\mathrm{Ca}^{+2}$ e bicarbonatos $\left(\mathrm{HCO}_{3}{ }^{-}\right)$ dissolvidos na água, sendo esta formação favorecida pela diminuição da pressão ou aumento da temperatura no reservatório. Durante a formação da calcita, há a liberação de $\mathrm{CO}_{2}$, que quando associado à água poderá formar o ácido carbônico $\left(\mathrm{H}_{2} \mathrm{CO}_{3}\right)$, ocasionando a corrosão dos componentes metálicos presentes na coluna (SHAMS EL DIN, 2002) como observado na amostra AM1 dada a presença da fase $\mathrm{FeOOH}$.

\section{CONCLUSÕES:}

Tomando como base os resultados de EFRX e DRX pode-se concluir que o material incrustado na parede interna da coluna de produção da amostra AM1 é uma incrustação tipo ferruginosa, resultante da ação corrosiva do $\mathrm{H}_{2} \mathrm{~S}$ comumente encontrada em poços de 
produção, em que sua origem pode está associada tanto à ação biogênica como à abiogênica.

$\mathrm{Na}$ análise da superfície da amostra AM1 com o MEV foi possível visualizar que a amostra possui uniformidade nos elementos químicos encontrados independentemente da região de analisada pelo EDS. O produto de corrosão Goetita $\left(\mathrm{Fe}^{+3} \mathrm{O}(\mathrm{OH})\right)$ é provavelmente originado da oxidação superficial do tubo, com posterior deposição dos produtos de corrosão, originados pela ação do $\mathrm{H}_{2} \mathrm{~S}$, sendo esta interface bem visível pela analise de MEV utilizando-se análise por BSE.

Já a amostra AM1 pode ser classificada como incrustação mista, ou seja, tem-se a deposição da lama argilosa (confirmada através da presença de alumínio e silício no EFRX da amostra AM1) juntamente com produtos de corrosão.

\section{AGRADECIMENTOS:}

Agradecemos ao CENPES/PETROBRAS (Centro de Pesquisas e Desenvolvimento Leopoldo A. Miguez de Mello) pelos investimentos em infraestrutura no NUPER/UFRN (Núcleo de Petróleo e Energias Renováveis/Universidade Federal do Rio Grande do Norte).

\section{REFERÊNCIAS:}

1. ATKINSON, G. MECIK, M. RAJU, K.U.G. HOWELL. Thermodynamics of Scale Mineral Solubilities: The Efect of Pressure. In: The NACE Annual Conference and Corrosion Show. Paper Number 31, Corrosion, 1992.

2. BOIM, J. M. Tese - Modelagem SemiAnalítica para Incrustação de Sulfato de Bário em Reservatórios Heterogêneos. Universidade Estadual do Norte Fluminense - UENF, Macaé/RJ - Brasil, Dezembro, 2003.

3. DAHER, J. S. Avaliação de Incrustação de Sais Inorgânicos em Reservatórios Inconsolidados Através da Simulação Numérica. 211f. Dissertação de Mestrado. Programa de Pós-Graduação em Engenharia de Reservatório e de Exploração de Petróleo, Universidade
Estadual do Norte Fluminense, 2003. (in Portuguese).

4. DEMADIS, K. D.; MAVREDAKI, E.; STATHOULOPOULOU, A.; NEOFOTISTOU, E.; MANTZARIDIS, C. Industrial water systems: problems, challenges and solutions for the process industries. Desalination, $v$. 213, p. 38-46, 2007.

5. DEARBORN, B. Tratamento de Água de Resfriamento. 9a ed. 1991.

6. GENTIL, V. Corrosão. Editora LTC. $5^{\mathrm{a}}$ ed. Rio de Janeiro/RJ - Brasil, 2007.

7. Hemmingsen, T.; Lima, $H$. Electrochemical and Optical Studies of Sulphide Film Formation on Carbon Steel. Electrochimica Acta, v. 43, p. 35-40, 1998.

8. MARQUES, L. C. C. PEREIRA, A. Z. I. MEIRELLES Jr., R. O. GONÇALVES, M. A. C. How Petrobras Has Faced Oilfield Scale Problems: Evolution of Concepts and Lessons Learned in Campos Basin, Brazil. In: SPE International Symposium on Oilfield Scale held, Aberdeen - UK, 30-31, Janeiro, 2001.

9. MOURA, D. M. A.; ARAÚJO, B. A. T.; MACHADO, S. F.; TÁVORA, M. P.; SILVA, A. A. R.; CUNHA, J. D.; SILVA, D. R. Eficiência de Solventes Orgânicos Apolares na Extração de Produtos de Corrosão. In: VI Corrosion Congress Nace International Latin America Region, 2006, Proceeding LatinCorr 2006, Fortaleza - CE, 1 CD-ROM.

10. NEAL, A. L.; TECHKARNJANARUK, S.; DOHNALKOVA, A.; MCCREADY, D.; PEYTON, B. M.; GEESEY, G. G. Iron sulfides and sulfur species produced at hematite surfaces in the presence of sulfate-reducing bacteria. Geochimica et Cosmochimica Acta, v. 65, No. 2, p. 223235, 2001.

11. RAMTAD, K.; TYDAL, T.; ASKVIK, K. M.; FOTLAND, P. Predicting Carbonate Scale in Oil Producers From High Temperature Reservoirs. SPE Journal, v. 10, No. 4, p. 363-373, 2005.

12. WOOD, R. J. K. Erosion-corrosion interactions and their effect on marine and offshore materials. Wear, v. 261, p. 10121023,2006 
Tabela 01 - Resultados do teor inorgânico $\left(T_{\text {inorg }}\right)$ e orgânico $\left(T_{\text {org }}\right)$ após extração Soxhlet.

\begin{tabular}{ccc} 
Amostra & $\mathbf{T}_{\text {inorg }}(\%)$ & $\mathbf{T}_{\text {org }}(\%)$ \\
\hline AM1 & 97,27 & 2,67 \\
\hline
\end{tabular}

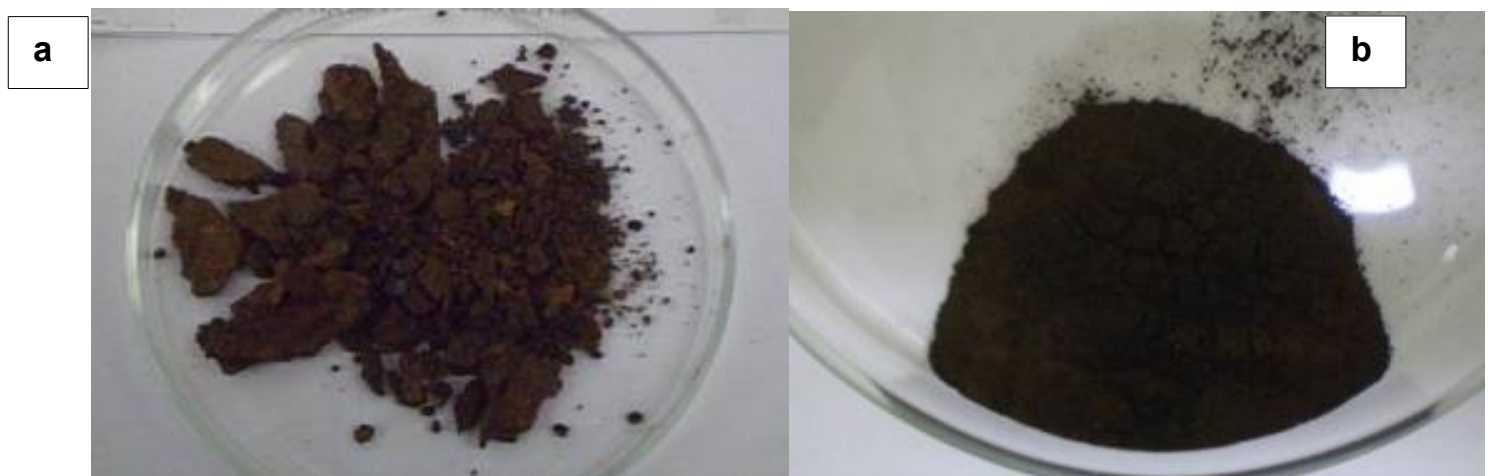

Figura 1 - a) aspecto da amostra AM1 antes da extração Soxhlet e b) amostra já extraída e pulverizada.

Tabela 02 - Resultado de EFRX (modo semi-quantitativo) referente à amostra AM1. Elemento \%

\begin{tabular}{|c|c|c|c|c|c|c|c|c|c|c|c|c|c|}
\hline & \\
\hline & $\mathrm{Al}$ & $\mathrm{Ca}$ & $\mathrm{Cl}$ & $\mathrm{Cu}$ & $\mathrm{Cr}$ & $S$ & $\mathrm{Sr}$ & $\mathrm{Fe}$ & $P$ & $\mathrm{Mn}$ & $\mathrm{Ni}$ & $\mathrm{K}$ & $\mathrm{Si}$ \\
\hline AM1 & 0,14 & 0,41 & 0,17 & ND & 0,07 & 18,36 & ND & 78,50 & 0,05 & 0,42 & ND & ND & 1,86 \\
\hline
\end{tabular}

ND - Não detectado

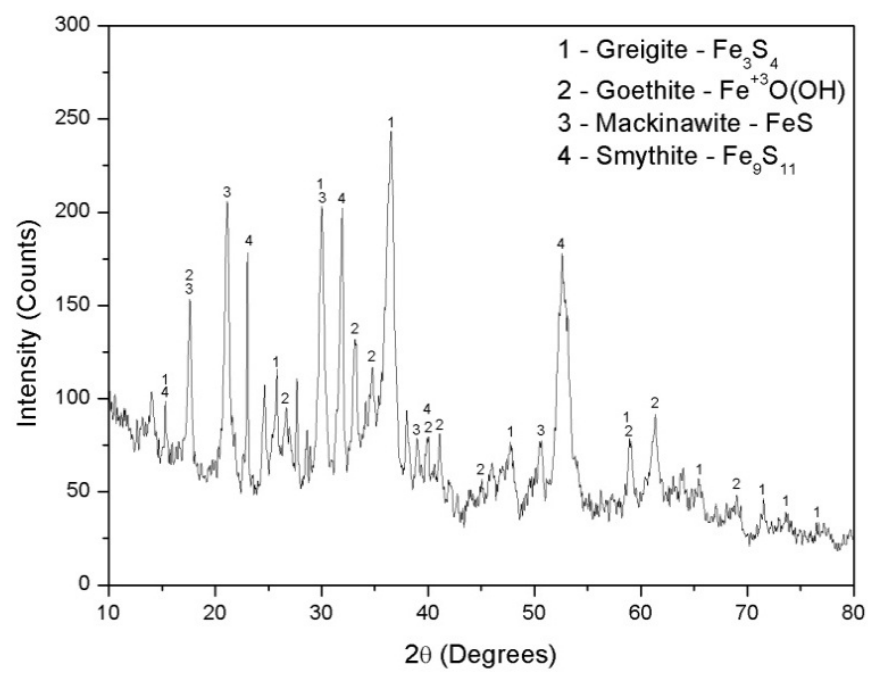

Figura 2 - Difratograma das principais fases presentes na amostra AM1.

PERIÓDICO TCHÊ QUÍMICA • www.periodico.tchequimica.com • Vol. 11 N. 22.

- ISSN 1806-0374 (impresso) • ISSN 1806-9827 (CD-ROM) • ISSN 2179-0302 (meio eletrônico)

(C) 2014. Porto Alegre, RS. Brasil 
Tabela 03 - Numeração das cartas referente às fases presentes na amostra AM1, segundo o banco de dados do JCPDS, e suas respectivas estruturas cristalinas.

\begin{tabular}{cccc}
\hline Nome do Mineral & Fórmula Química & JCPDS & Estrutura Cristalina \\
\hline Greigita & $\mathrm{Fe}_{3} \mathrm{~S}_{4}$ & $16-0713$ & Cubica de face-centrada \\
Goetita & $\mathrm{Fe}^{+3} \mathrm{O}(\mathrm{OH})$ & $29-0713$ & Orthorhombica \\
Mackinawita & $\mathrm{FeS}$ & $15-0037$ & Tetragonal \\
Smythita & $\mathrm{Fe}_{9} \mathrm{~S}_{11}$ & $10-0437$ & Rhombohedral rhomb-centered \\
\hline
\end{tabular}
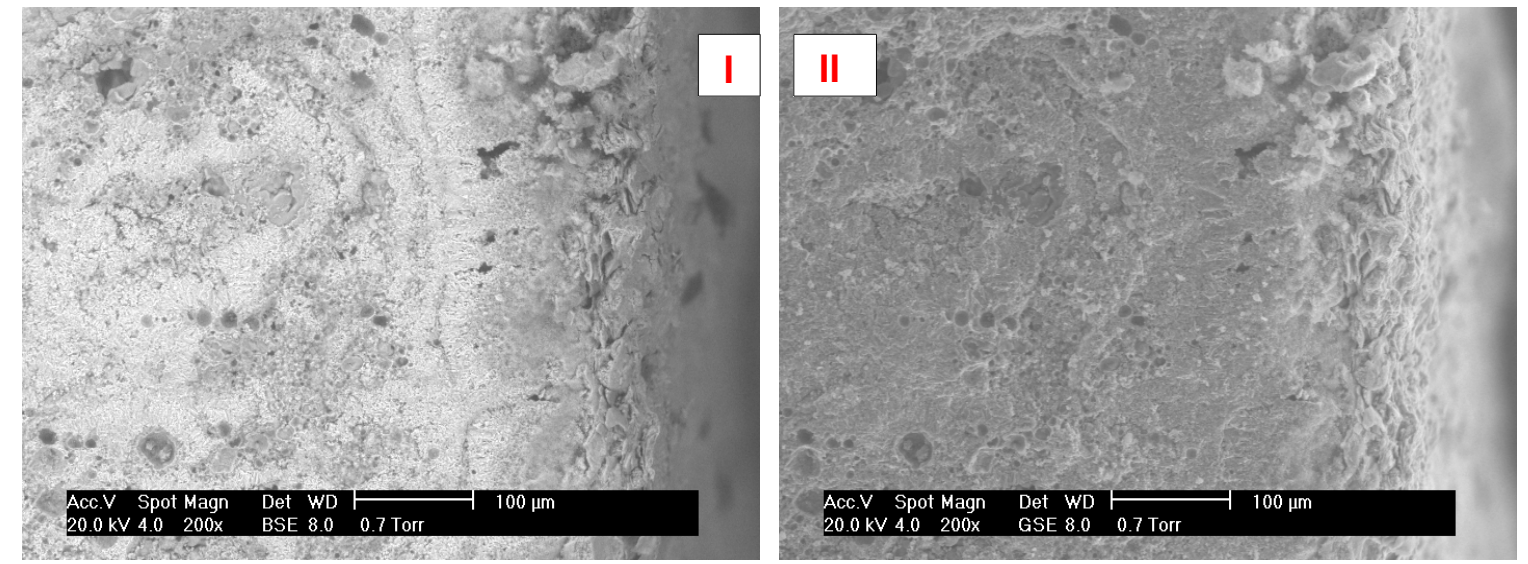

Figura 5- MEV da amostra AM1. Região de contato com o tubo. I - BSE com os pontos nos quais foram realizadas as análises químicas por EDS. II - SE no qual é observada a morfologia da região.

Tabela 6- Composição química dos pontos marcados na Figura 5-I, nos quais foram realizadas as microanálises químicas por EDS.

\begin{tabular}{cccccc}
\hline \multirow{2}{*}{ Elemento } & \multicolumn{5}{c}{ Composição (\%) } \\
& Ponto-A & Ponto-B & Ponto-C & Ponto-D & Ponto-E \\
\hline $\mathrm{C}$ & 5,12 & 5,27 & 2,68 & 3,84 & 9,27 \\
$\mathrm{O}$ & 18,16 & 7,00 & 8,74 & 6,61 & 15,54 \\
$\mathrm{Al}$ & 10,21 & 6,83 & 5,78 & 7,22 & 6,19 \\
$\mathrm{Si}$ & 1,69 & 0,94 & 1,00 & 0,00 & 0,00 \\
$\mathrm{~S}$ & 4,64 & 19,78 & 41,09 & 21,78 & 28,43 \\
$\mathrm{Ca}$ & 0,55 & 0,43 & 0,30 & 0,00 & 0,00 \\
$\mathrm{Fe}$ & 59,63 & 59,75 & 40,41 & 60,56 & 40,57 \\
\hline
\end{tabular}

PERIÓDICO TCHÊ QUÍMICA • www.periodico.tchequimica.com • Vol. 11 N. 22.

• ISSN 1806-0374 (impresso) • ISSN 1806-9827 (CD-ROM) • ISSN 2179-0302 (meio eletrônico)

(C) 2014. Porto Alegre, RS. Brasil

The Periódico Tchê Química (ISSN: 1806-0374; 2179-0302) is an open-access journal since 2004. Journal DOI: 10.52571/PTQ. http://www.tchequimica.com. This text was introduced in this file in 2021 for compliance reasons.

() The Author(s)
OPEN ACCESS. This article is licensed under a Creative Commons Attribution 4.0 (CC BY 4.0) International License, which permits use, sharing, adaptation, distribution, and reproduction in any medium or format, as long as you give appropriate credit to the original author(s) and the source, provide a link to the Creative Commons license, and indicate if changes were made. The images or other third-party material in this article are included in the or exceeds the permitted use, you will need to obtain permission directly from the copyright holder. To view a copy of this license, visit http://creativecommons. org/licenses/by/4.0/. 